Osteoporotic Fractures (SOF; participants were aged 65 years or older at baseline). These patients were compared with 1,317 controls; all individuals were genotyped for the Arg200Trp and Arg324Gly variants of FRP. They found that women homozygous for the Arg200Trp allele of FRP had three times the risk of developing radiographically visible osteophytes in the femoral head, compared with controls. The Arg324Gly mutation was associated with increased risk of radiographically visible jointspace narrowing, an association that was markedly enhanced when both mutations were present.

The authors speculate that individuals with the Arg324Gly mutation might experience accelerated progression of hip OA. They note that the low frequency of these FRPB mutations in SOF participants might be because individuals with bilateral hip replacements were excluded from the SOF cohort, which could have excluded women with risk-conferring genotypes, and call for further studies to confirm the association of joint-space narrowing with the Arg324Gly genotype.

Original article Lane NE et al. (2006) Frizzled-related protein variants are risk factors for hip osteoarthritis. Arthritis Rheum 54: 1246-1254

\section{Low estrogen levels are implicated in pathogenesis of giant cell arteritis}

Results of a case-control study in Sweden suggest that pathogenesis of the chronic, inflammatory disorder giant cell arteritis (which has a high incidence in Scandinavian ethnic groups), is affected by levels of female sex hormones. Larsson and colleagues found that low BMI, menopause before the age of 43 , smoking, and longer duration of breast feeding were all independent risk factors associated with giant cell arteritis.

The authors identified 65 cases of biopsyproven giant cell arteritis in women aged 50-69 years at diagnosis; the 49 women who responded to a questionnaire on hormonal and reproductive factors were included in the study. Controls were 10,405 women who had responded to the same questionnaire as part of a Swedish national breast-cancer prevention program.
Giant cell arteritis mainly affects postmenopausal women, who experience an agerelated decline in production of adrenal steroids and gonadal hormones. Larsson and colleagues suggest that extended periods of low production of female sex hormones suppress the hypothalamic-pituitary-gonadal axis and blunt the responsiveness of the hypothalamicpituitary-adrenal axis, thereby exacerbating inflammatory processes.

Postmenopausal women with low BMI have high levels of sex-hormone-binding globulin and low levels of estrone (the principal estrogen in postmenopausal women, which is produced mainly in adipose tissue). Interestingly, smoking is known to reduce BMI and decrease estrone synthesis, and is also associated with early onset of menopause, but was an independent risk factor for giant cell arteritis in this study.

Original article Larsson K et al. (2006) Early menopause, low body mass index, and smoking are independent risk factors for developing giant cell arteritis. Ann Rheum Dis 65: $529-532$

\section{Vitamin $\mathrm{K}$ deficiency is associated with radiographically visible features of osteoarthritis}

Inadequate levels of vitamin-K-dependent proteins are associated with abnormal mineralization of bone and cartilage in animal models, indicating important roles for this vitamin in regulation of bone and cartilage metabolism, and in growth-plate calcification. Serum levels of phylloquinone (the principal dietary form of vitamin $\mathrm{K}$, obtained from green leafy vegetables) reflect short-term vitamin K intake; Neogi et al. investigated whether plasma phylloquinone levels were associated with radiographically visible features of osteoarthritis $(\mathrm{OA})$ in the knee and hand. They found that low vitamin $\mathrm{K}$ status was associated with joint-space narrowing and $\mathrm{OA}$ in the hand, and with the presence of large osteophytes in both the hand and the knee.

They analysed data from 672 participants in the Framingham Offspring Study (mean age 66 years), whose plasma phylloquinone levels were measured in 1996-1998, and who underwent bilateral hand and knee radiography in 2002-2004. Median plasma phylloquinone in the study group was $1.03 \mathrm{nmol} / \mathrm{l}$ (normal range, $0.5-2.5 \mathrm{nmol} / \mathrm{l})$; about half of the cohort had low 